\title{
Phenotype, genotype and long-term prognosis of 40 Chinese patients with isobutyryl-CoA dehydrogenase deficiency and a review of variant spectra in $A C A D 8$
}

Junqi Feng ${ }^{1,2+}{ }^{\text {, Chenxi Yang }}{ }^{2+}$, Ling Zhu ${ }^{1}$, Yuchen Zhang ${ }^{2}$, Xiaoxu Zhao ${ }^{1}$, Chi Chen ${ }^{1}$, Qi-xing Chen ${ }^{1}$, Qiang Shu ${ }^{\text {* }}$, Pingping Jiang ${ }^{1,2,3^{*}}$ (ID and Fan Tong ${ }^{1 *}$

\begin{abstract}
Background: Isobutyryl-COA dehydrogenase deficiency (IBDD) is a rare autosomal recessive metabolic disorder resulting from variants in $A C A D 8$, and is poorly understood, as only dozens of cases have been reported previously. Based on a newborn screening program, we evaluated the incidence, phenotype and genotype of IBDD as well as the prognosis. Moreover, we reviewed the variant spectrum in ACAD8 associated with IBDD.

Methods: Forty unrelated patients with IBDD were retrospectively screened for newborns between Jan 2012 and Dec 2020. Tandem mass spectrometry (MS/MS) was used to determine the concentrations of C4-acylcarnitine, C4/C2 (acetylcarnitine), and C4/C3 (propionylcarnitine). All suspected cases were genetically tested by metabolic genes panel.

Results: The incidence of IBDD here was 1: 62,599. All patients presented continuously elevated C4-acylcarnitine levels with higher ratios of $\mathrm{C} 4 / \mathrm{C} 2$ and $\mathrm{C} 4 / \mathrm{C} 3$. Isobutyrylglycine occurred in only 8 patients. During follow-up, four patients had a transient motor delay, and two patients had growth delay. Notably, one case harbored both ACAD8 compound heterozygous variants and a KMT2A de novo variant (c.2739del, p.E914Rfs*35), with IBDD and Wiedemann-Steiner syndrome together, had exact severe global developmental delay. All patients were regularly monitored once they were diagnosed, and each patient gradually had a normal diet after 6 months of age. After 3-108 months of follow-up, most individuals were healthy except the case harboring the KMT2A variant. A total of 16 novel variants in ACAD8, c.4_5delCT, c.109C > T, c.110-2A>T, c.236G > A, c.259G > A, c.381-14G > A, c.413delA, c.473A > G, c.500delG, c.758 T>G, c.842-1G >A, c.911A>T, c.989G >A, c.1150G >C, c.1157A > G and c.1165C > T, were identified. Along with a literature review on 51 ACAD 8 variants in 81 IBDD patients, we found that the most common
\end{abstract}

\footnotetext{
*Correspondence: shuqiang@zju.edu.cn; ppjiang@zju.edu.cn; 194008@zju.

edu.cn

†Junqi Feng and Chenxi Yang have contributed equally to this work

${ }^{1}$ Department of Genetic and Metabolic Disease, The Children's Hospital,

Zhejiang University School of Medicine, National Clinical Research Center

for Child Health, 3333 Binsheng Road, Hangzhou 310052, China

${ }^{2}$ Institute of Genetics and Department of Human Genetics, Zhejiang

University School of Medicine, Hangzhou 310058, China

Full list of author information is available at the end of the article
}

(c) The Author(s) 2021, corrected publication 2021. Open Access This article is licensed under a Creative Commons Attribution 4.0 International License, which permits use, sharing, adaptation, distribution and reproduction in any medium or format, as long as you give appropriate credit to the original author(s) and the source, provide a link to the Creative Commons licence, and indicate if changes were made. The images or other third party material in this article are included in the article's Creative Commons licence, unless indicated otherwise in a credit line to the material. If material is not included in the article's Creative Commons licence and your intended use is not permitted by statutory regulation or exceeds the permitted use, you will need to obtain permission directly from the copyright holder. To view a copy of this licence, visit http://creativecommons.org/licenses/by/4.0/. The Creative Commons Public Domain Dedication waiver (http:/creativecommons.org/publicdomain/zero/1.0/) applies to the data made available in this article, unless otherwise stated in a credit line to the data. 
variant was c.286G > A (27.2\%), which has been observed solely in the Chinese population to date, followed by c. $1000 \mathrm{C}>\mathrm{T}(8.6 \%), \mathrm{c} .1176 \mathrm{G}>\mathrm{T}$ (3.7\%) and c.455 T > C (3.1\%).

Conclusion: The concentration of C4-acylcarnitine in NBS plus subsequent genetic testing is necessary for IBDD diagnosis. Both the genotypes and ACAD8 variants in IBDD are highly heterogeneous, and no significant correlations between genotype and phenotype are present here in patients with IBDD. Our IBDD cohort with detaied clinical characteristics, genotypes and long-term prognosis will be helpful for the diagnosis and management of patients with IBDD in the future.

Keywords: Isobutyryl-CoA dehydrogenase deficiency (IBDD), ACAD8, KMT2A, Genotype, Phenotype, Prognosis

\section{Background}

Isobutyryl-CoA dehydrogenase (IBD) deficiency (IBDD, OMIM\#611283) is a rare autosomal recessive metabolic disorder involving defects in valine metabolism [1], which is caused by biallelic variants in ACAD8 (acyl-CoA dehydrogenase family member 8; OMIM*604773) on chromosome 11q25 [2]. IBD is a mitochondrial enzyme that functions as a soluble homotetramer to catalyse the conversion of isobutyryl-CoA to methacrylyl-CoA in the degradation of the branched chain amino acid (BCAA) 'valine,' which finally converges on the tricarboxylic acid cycle. To date, only dozens of IBDD cases have been reported worldwide since it was first described in 1998, with an estimated prevalence of $1 / 45,466$ in southern Italy and 1/292,451 in California [3, 4]. Affected individuals are mostly diagnosed through newborn screening (NBS) and characterized by elevated C4-acylcarnitine levels, with or without secondary carnitine deficiency [1, 5-8]. However, elevated urine isobutyrylglycine (IBG) levels have been documented as additional supporting evidence, but this change is not always present in patients with IBDD [9]. Patients with IBDD are reported to be either asymptomatic $[5,10]$, or symptomatic with variable clinical features, including failure to thrive, seizures, anaemia, muscular hypotonia, and developmental delay $[5-9,11,12]$. Some symptomatic patients had normal growth and development with carnitine supplementation and avoidance of fasting to limit amino acid metabolism during childhood [5]. In contrast, one asymptomatic child with IBDD was reported to develop clinical symptoms, including muscle pain, muscle weakness and tiredness, in adulthood [13], indicating a complicated pathogenesis and suggesting that patients with IBDD should be monitored carefully [14]. As elevated C4-acylcarnitine concentrations are neither specific nor sufficient for the clinical diagnosis of IBDD, genetic testing is increasingly applied for the identification of IBDD. Recently, more than 30 variants in ACAD8 have been reported in different ethnic populations. However, the clinical, genetic and prognostic data of IBDD are limited by the number of cases. Here, we retrospectively summarized the phenotypes, genotypes and long-term prognosis of up to 40 patients with IBDD identified between 2012 and 2020, and reviewed all ACAD8 variants in described IBDD cases, including 16 novel variants in this study.

\section{Materials and methods \\ Subjects}

Forty patients with a diagnosis of IBDD based on the NBS program within 7 days after birth were retrospectively reviewed between 2012 and December 2020 in a single site newborn screening program, among which 30 patients were identified from 1,877,970 infants between January 2017 and December 2020. This study was conducted at the Children's Hospital, Zhejiang University School of Medicine, and approved by the Research Ethics Committees. Patient information was tabulated in this article without individual patient identifiers.

\section{Metabolic marks analysis}

Tandem mass spectrometry (MS/MS) was used to determine the concentrations of C4-acylcarnitine, C4/C2 (acetylcarnitine), and $\mathrm{C} 4 / \mathrm{C} 3$ (propionylcarnitine) with dried blood spot filter paper samples in the NBS program. Urine samples were collected from the patients for a urine organic acid analysis by gas chromatographmass spectrometer (GC-MS). The following cut-off parameters in our clinical lab were used for validation: normal values of C4-acylcarnitine ranged from 0.03 to $0.48(\mu \mathrm{mol} / \mathrm{L})$ and those of urinary IBG ranged from 0 to $0.4(\mathrm{mmol} / \mathrm{mol})$. Moreover, the normal $\mathrm{C} 4 / \mathrm{C} 2$ ratio decreased in the $0-0.05$ interval, and the $\mathrm{C} 4 / \mathrm{C} 3$ ratio decreased in the 0.04-0.46 interval. The Wechsler Intelligence Scale for Children-R (WISC-R) or the AgesStages Questionnaire (ASQ) were used to assess patients' developmental status. Length/height-for-age and weightfor-age standards are according to China growth standards $(0-3$ years old) and WHO Child Growth Standards ( $>3$ years old).

\section{Genetic analysis}

Triometabolic gene panel tests by NGS (next-generation sequencing) were conducted in all suspected cases who were picked up by NBS, including the $A C A D 8, A C A D S$, 
ETHE1, ETFA, ETFB, and ETFDH genes. The DNA library was prepared using an Agilent SureSelect Inherited Disease Capture Kit (Agilent, USA) and sequenced using an Illumina MiSeq platform (Illumina, USA). The quality of the DNA library was tested with a Qubit and 2100 Bioanalyzer (Agilent High Sensitivity DNA Kit, Agilent). The sequencing libraries were quantified using an Illumina DNA Standards and Primer Premix Kit (Kapa, USA). The paired-end reads were quality trimmed using the Trimmomatic program (http://www.usadellab.org/

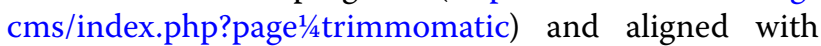
the human genome reference sequence (UCSC Genome build hg19). Single-nucleotide polymorphisms (SNPs) and insertions or deletions were identified using the Samtools program (http://www.htslib.org/). The ClinVar and Human Gene Mutation Database (HGMD) was used to search for known pathogenic mutations. Automatic tools (SIFT, Polyphen, MutationTaster, CADD, etc.) were used to predict the functional significance of novel variants. Variants were classified according to the recommendations of the American College of Medical Genetics and Genomics (ACMG) as follows: pathogenic (P), likely pathogenic (LP), variants of unknown significance (VUS), likely benign (LB), and benign (B) [15].

\section{Results}

\section{Clinical manifestations and interventions}

A cohort of 40 unrelated patients with IBDD is summarized in Table 1. The IBDD incidence was 1: 62,599 based on data from the NBS program between 01/2017 and $12 / 2020$. In this cohort, patients \#9 and \#22 were born prematurely, but their mothers' pregnancies were uneventful to that point, and they did not require any significant interventions. The remaining 38 patients were born after uneventful pregnancies at full term. All 40 patients were evaluated for significantly increased concentrations of C4-acylcarnitine ranging from 0.98 to $3.36 \mu \mathrm{mol} / \mathrm{L}$ and elevated $\mathrm{C} 4 / \mathrm{C} 2$ and $\mathrm{C} 4 / \mathrm{C} 3$ ratios by NBS. Consistent with previous documents, isobutyrylglycine occurred in only 8 patients (patients \#2, \#11, \#13, $\# 16$, \#21, \#27, \#35 and \#38) from the cohort. Ten patients (patients \#1, \#12, \#19, \#25, \#27, \#29, \#31, \#36, \#39 and $\# 40)$ had anaemia as described elsewhere [10]. Some biochemical indicators associated with liver function, such as aminotransferase and gamma-glutamyl transferase, were aberrant in 18 patients from 0.5-7 months after birth. However, the relationship between abnormal liver function and IBDD is not clear as abnormal liver function is common in most children patients with inherited metabolic disease, especially in infants. Regarding development, patients \#9, \#10, \#12 and \#14 experienced a transient motor delay, and all recovered during followup. However, patient \#33 had a severe developmental delay, with an FIQ of 53 according to WISC-R. Subsequently, by whole exome sequencing and analysis, a de novo variant c.2739del (p.E914Rfs*35) in KMT2A was detected in patient \#33, which was absent in gnomAD (Genome Aggregation Database). As KMT2A is known as the causative gene in Wiedemann-Steiner syndrome (WDSTS, OMIM\#605130), this truncating variant was implied to be responsible for the manifestations of severe growth and developmental delay in patient \#33. The height/length-for-age and weight-for-age of patients \#5, $\# 15, \# 17$, \#19 and \#22 were slightly delayed during follow-up. Patient \#25, who with milk allergy, and patient \#33 had severe thriving failure.

Consistent with published cases, most patients with IBDD turned out healthy and had no clinical sequelae $[6,10,16]$. Once diagnosed, all patients with IBDD were treated with sufficient caloric supplementation and dietary high-protein restriction (protein intake: $2-2.5 \mathrm{~g} / \mathrm{kg} / \mathrm{d}$ ) to avoid fasting and avoid protein catabolism. Under the guidance of medical specialists, infants younger than 6 months were nourished with full breastfeeding or formula supplementation when breast milk was insufficient. Elder infants were nourished additionally with introduced complementary foods to meet daily protein requirements. The feeding interval for infants less than 6 months was no more than $4 \mathrm{~h}$, that for infants 6-12 months was no more than $6-8 \mathrm{~h}$, that for infants $1-3$ years was no more than $8-10 \mathrm{~h}$, and that for infants 3 years and above was no more than 10-12 h. Patient \#33 accepted L-carnitine $(50 \mathrm{mg} / \mathrm{kg} / \mathrm{d})$ and vitamin B2 $(100 \mathrm{mg} / \mathrm{d})$ supplementation for one year and then stopped since there was no improvement in clinical symptoms or biochemical indictors. Patient \#25 was diagnosed with cow's milk protein allergy (CMPA) by a gastroenterologist, and then his complementary food was changed to extensively hydrolysed formula, which has insufficient calories to supply and may account for his growth delay during infancy. Fortunately, his growth delay was improved later with the diet transitioning to toddler nutrition. Moreover, each parent had been educated and reminded to introduce complementary foods as stated and then gradually transitioned to normal toddler nutrition after 1.5-2 years of age. After 6 months of follow-up, each patient gradually had a normal diet. During follow-up, each patient's height/length, weight and health were regularly monitored. Assisted by familial rehabilitation, no other metabolic imbalance occurred, while C4-acylcarnitine was still sustained at high concentrations (Additional file 1: Table S2).

\section{Genetic variants and genotypes}

Biallelic variants were genotyped in 39 individuals except patient \#7, in which only one variant was detected 
Table 1 Genotypes and clinical features in 40 IBDD cases

\begin{tabular}{|c|c|c|c|c|c|c|c|c|c|}
\hline \multirow[t]{2}{*}{ Case } & \multicolumn{2}{|l|}{ Genotype } & \multirow{2}{*}{$\begin{array}{l}\text { Pathogenicity } \\
\text { classification }\end{array}$} & \multicolumn{2}{|c|}{ CADD Score } & \multicolumn{3}{|l|}{ NBS } & \multirow{2}{*}{$\begin{array}{l}\text { Clinical } \\
\text { symptom }\end{array}$} \\
\hline & Allele 1 & Allele 2 & & Allele 1 & Allele 2 & $\begin{array}{l}C 4 \\
(0.03- \\
0.48 \mu \mathrm{mol} / \mathrm{L})\end{array}$ & $\begin{array}{l}C 4 / C 2 \\
(0-0.05)\end{array}$ & $\begin{array}{l}\text { C4/C3 } \\
(0.04-0.46)\end{array}$ & \\
\hline 1 & $\begin{array}{l}\text { c.4_5delCT } T^{\#} \\
\left(p . L 2 V f s^{*} 40\right)\end{array}$ & c.842-1G > A \# & $\mathrm{P} / \mathrm{P}$ & 20.7 & 31 & $1.86 \uparrow$ & $0.16 \uparrow$ & $1.14 \uparrow$ & (4) \\
\hline 2 & $\begin{array}{l}\text { c.286G >A } \\
\text { (p.G96S) }\end{array}$ & $\begin{array}{l}\text { c.286G >A } \\
\text { (p.G96S) }\end{array}$ & $L P / L P$ & 30 & 30 & $1.79 \uparrow$ & $0.11 \uparrow$ & $1.28 \uparrow$ & (3) \\
\hline 3 & $\begin{array}{l}c .286 G>A \\
\text { (p.G96S) }\end{array}$ & $\begin{array}{l}\text { c.1176G >T } \\
\text { (p.R392S) }\end{array}$ & $L P / L P$ & 30 & 25.5 & $1.5 \uparrow$ & $0.11 \uparrow$ & $1.02 \uparrow$ & - \\
\hline 4 & $\begin{array}{l}c .1000 C>T \\
(p . R 334 C)\end{array}$ & $\begin{array}{l}\text { c. } 235 C>G \\
(p . R 79 G)\end{array}$ & $L P / L P$ & 27.2 & 24.7 & $1.2 \uparrow$ & $0.1 \uparrow$ & $1.5 \uparrow$ & - \\
\hline 5 & $\begin{array}{l}\text { c.286G >A } \\
\text { (p.G96S) }\end{array}$ & $\begin{array}{l}\text { c.286G >A } \\
\text { (p.G96S) }\end{array}$ & $L P / L P$ & 30 & 30 & $1.24 \uparrow$ & 0.05 & $0.98 \uparrow$ & (4) \\
\hline 6 & $\begin{array}{l}\text { c.286G > A } \\
\text { (p.G96S) }\end{array}$ & $\begin{array}{l}\text { c.911A > } T^{\#} \\
(\text { p.Q304L) }\end{array}$ & LPNUS & 30 & 31 & $1.57 \uparrow$ & $0.13 \uparrow$ & $0.86 \uparrow$ & - \\
\hline 7 & $\begin{array}{l}\text { c.286G >A } \\
\text { (p.G96S) }\end{array}$ & - & $\mathrm{LP} / ?$ & 30 & - & $1.06 \uparrow$ & $0.07 \uparrow$ & $0.7 \uparrow$ & (4) \\
\hline 8 & $\begin{array}{l}\text { c.286G >A } \\
\text { (p.G96S) }\end{array}$ & $\begin{array}{l}\text { c.455 T>C } \\
\text { (p.M152T) }\end{array}$ & $L P / L P$ & 30 & 27.4 & $2.55 \uparrow$ & $0.18 \uparrow$ & $2.48 \uparrow$ & - \\
\hline 9 & $\begin{array}{l}\text { c.286G >A } \\
\text { (p.G96S) }\end{array}$ & $\begin{array}{l}\text { c.712delT } \\
\text { p.W238 fs*9 }\end{array}$ & $L P / P$ & 30 & 35 & $1.66 \uparrow$ & $0.21 \uparrow$ & $3.07 \uparrow$ & (2) \\
\hline 10 & $\begin{array}{l}\text { c.286G >A } \\
\text { (p.G96S) }\end{array}$ & $\begin{array}{l}\text { c.286G >A } \\
\text { (p.G96S) }\end{array}$ & $L P / L P$ & 30 & 30 & $1.66 \uparrow$ & $0.1 \uparrow$ & $1.71 \uparrow$ & (2)(4) \\
\hline 11 & $\begin{array}{l}c .1000 C>T \\
(p . R 334 C)\end{array}$ & $\begin{array}{l}\text { c.286G > A } \\
\text { (p.G96S) }\end{array}$ & $L P / L P$ & 27.2 & 30 & $1.86 \uparrow$ & $0.15 \uparrow$ & $1.84 \uparrow$ & (3) (4) \\
\hline 12 & $\begin{array}{l}\text { c.1000C > T } \\
(p . R 334 C)\end{array}$ & $\begin{array}{l}\text { c.286G >A } \\
\text { (p.G96S) }\end{array}$ & $L P / L P$ & 27.2 & 30 & $2.02 \uparrow$ & $0.09 \uparrow$ & $1.64 \uparrow$ & (2) (4) \\
\hline 13 & $\begin{array}{l}\text { c.286G > A } \\
\text { (p.G96S) }\end{array}$ & $\begin{array}{l}\text { c.235C > G } \\
(p . R 79 G)\end{array}$ & LP/LP & 30 & 24.7 & $2.88 \uparrow$ & $0.13 \uparrow$ & $1.18 \uparrow$ & (3) (4) \\
\hline 14 & $\begin{array}{l}\text { c.286G > A } \\
\text { (p.G96S) }\end{array}$ & $\begin{array}{l}\text { c.444G > T } \\
\text { (p.P148P) }\end{array}$ & $L P / L B$ & 30 & 4.199 & $1.16 \uparrow$ & 0.04 & $0.56 \uparrow$ & (2) \\
\hline 15 & $\begin{array}{l}\text { c.286G > A } \\
\text { (p.G96S) }\end{array}$ & $\begin{array}{l}\text { c.413delA }{ }^{\#} \\
\text { (p.N138Mfs*36) }\end{array}$ & $L P / P$ & 30 & 23.4 & $1.67 \uparrow$ & $0.1 \uparrow$ & $1.01 \uparrow$ & (4) \\
\hline 16 & $\begin{array}{l}\text { c.286G >A } \\
\text { (p.G96S) }\end{array}$ & $\begin{array}{l}\text { c.286G >A } \\
\text { (p.G96S) }\end{array}$ & $L P / L P$ & 30 & 30 & $2.96 \uparrow$ & $0.11 \uparrow$ & $1.55 \uparrow$ & (3) \\
\hline 17 & $\begin{array}{l}\text { c.413delA }{ }^{\#} \\
\text { (p.N138Mfs*36) }\end{array}$ & $\begin{array}{l}\text { c.500delG }{ }^{\#} \\
(\text { p.S167Mfs*7) }\end{array}$ & $\mathrm{P} / \mathrm{P}$ & 23.4 & 25.3 & $1.59 \uparrow$ & $0.11 \uparrow$ & $1.92 \uparrow$ & - \\
\hline 18 & $\begin{array}{l}\text { c.286G >A } \\
\text { (p.G96S) }\end{array}$ & $\begin{array}{l}\text { c. } 286 G>A \\
\text { (p.G96S) }\end{array}$ & $L P / L P$ & 30 & 30 & $1.8 \uparrow$ & $0.09 \uparrow$ & $0.81 \uparrow$ & - \\
\hline 19 & c. $110-2 A>T^{\#}$ & $\begin{array}{l}\text { c.109C >T\# } \\
\text { (p.P37S) }\end{array}$ & $P / L P$ & 32 & 23.8 & $1.86 \uparrow$ & $0.1 \uparrow$ & $2.21 \uparrow$ & (4) \\
\hline 20 & $\begin{array}{l}c .1176 \mathrm{G}>\mathrm{T} \\
(\mathrm{p} . \mathrm{R} 392 \mathrm{~S})\end{array}$ & $\begin{array}{l}\text { c.444G > T } \\
\text { (p.P148P) }\end{array}$ & $L P / L B$ & 25.5 & 4.199 & $0.98 \uparrow$ & 0.05 & $0.58 \uparrow$ & - \\
\hline 21 & $\begin{array}{l}\text { c.286G >A } \\
\text { (p.G96S) }\end{array}$ & $\begin{array}{l}\text { c.286G >A } \\
\text { (p.G96S) }\end{array}$ & LP/LP & 30 & 30 & $1.43 \uparrow$ & $0.08 \uparrow$ & $0.88 \uparrow$ & (3) (4) \\
\hline 22 & $\begin{array}{l}c .259 G>A^{\#} \\
(p . G 87 R)\end{array}$ & $\begin{array}{l}\text { c. } 1000 C>T \\
(p . R 334 C)\end{array}$ & VUS/LP & 27.1 & 27.2 & $1.9 \uparrow$ & $0.23 \uparrow$ & $2.84 \uparrow$ & (4) \\
\hline 23 & $\begin{array}{l}\text { c.500delG\# } \\
\text { (p.S167Mfs*7) }\end{array}$ & $\begin{array}{l}\text { c.758 T> G\# } \\
(\text { p.V253G) }\end{array}$ & PNUS & 25.3 & 28.5 & $3.36 \uparrow$ & $0.12 \uparrow$ & $0.85 \uparrow$ & - \\
\hline
\end{tabular}


Table 1 (continued)

\begin{tabular}{|c|c|c|c|c|c|c|c|c|c|}
\hline \multirow[t]{2}{*}{ Case } & \multicolumn{2}{|l|}{ Genotype } & \multirow{2}{*}{$\begin{array}{l}\text { Pathogenicity } \\
\text { classification }\end{array}$} & \multicolumn{2}{|c|}{ CADD Score } & \multicolumn{3}{|l|}{ NBS } & \multirow{2}{*}{$\begin{array}{l}\text { Clinical } \\
\text { symptom }\end{array}$} \\
\hline & Allele 1 & Allele 2 & & Allele 1 & Allele 2 & $\begin{array}{l}\mathrm{C4} \\
(0.03- \\
0.48 \mu \mathrm{mol} / \mathrm{L})\end{array}$ & $\begin{array}{l}\mathrm{C} 4 / \mathrm{C} 2 \\
(0-0.05)\end{array}$ & $\begin{array}{l}\mathrm{C} 4 / \mathrm{C} 3 \\
(0.04-0.46)\end{array}$ & \\
\hline \multirow[t]{2}{*}{24} & C.1176G $>T$ & c. $1176 \mathrm{G}>\mathrm{T}$ & LP/LP & 25.5 & 25.5 & $2.33 \uparrow$ & $0.07 \uparrow$ & $1.08 \uparrow$ & - \\
\hline & (p.R392S) & (p.R392S) & & & & & & & \\
\hline \multirow[t]{2}{*}{25} & c. $1157 \mathrm{~A}>\mathrm{G}^{\#}$ & c. $1000 C>T$ & VUS/LP & 23.8 & 27.2 & $1.09 \uparrow$ & $0.07 \uparrow$ & $1.38 \uparrow$ & (1)(4) \\
\hline & (p.Q386R) & (p.R344C) & & & & & & & \\
\hline \multirow[t]{2}{*}{26} & c.4_5delCT\# & C.617G $>A$ & $\mathrm{P} / \mathrm{LP}$ & 20.7 & 30 & $2.29 \uparrow$ & $0.11 \uparrow$ & $1.89 \uparrow$ & - \\
\hline & $\left(p . L 2 V f s^{*} 39\right)$ & (p.R206Q) & & & & & & & \\
\hline \multirow[t]{2}{*}{27} & C. $989 G>A^{\#}$ & c.381-14G $>A^{\#}$ & LPNUS & 27.9 & 6.957 & $1.59 \uparrow$ & $0.08 \uparrow$ & $1.31 \uparrow$ & (3)(4) \\
\hline & (p.R330Q) & & & & & & & & \\
\hline \multirow[t]{2}{*}{28} & c. $1000 C>T$ & c. $617 \mathrm{G}>\mathrm{A}$ & $L P / L P$ & 27.2 & 30 & $2.57 \uparrow$ & $0.14 \uparrow$ & $1.82 \uparrow$ & (4) \\
\hline & (p.R344C) & (p.R206Q) & & & & & & & \\
\hline \multirow[t]{2}{*}{29} & $c .286 G>A$ & c. $286 G>A$ & $L P / L P$ & 30 & 30 & $1.64 \uparrow$ & $0.06 \uparrow$ & $1.39 \uparrow$ & (4) \\
\hline & (p.G96S) & (p.G96S) & & & & & & & \\
\hline \multirow[t]{2}{*}{30} & c. $286 \mathrm{G}>\mathrm{A}$ & c. $286 G>A$ & $L P / L P$ & 30 & 30 & $2.09 \uparrow$ & $0.14 \uparrow$ & $1.53 \uparrow$ & - \\
\hline & (p.G96S) & (p.G96S) & & & & & & & \\
\hline \multirow[t]{2}{*}{31} & $c .236 G>A^{\#}$ & c. $286 G>A$ & $L P / L P$ & 27.2 & 30 & $0.99 \uparrow$ & $0.13 \uparrow$ & $1.15 \uparrow$ & (4) \\
\hline & (P.R79Q) & (p.G96S) & & & & & & & \\
\hline \multirow[t]{2}{*}{32} & c.413delA\# & c. $286 G>A$ & $P / L P$ & 23.4 & 30 & $1.83 \uparrow$ & $0.29 \uparrow$ & $3.33 \uparrow$ & (4) \\
\hline & (p.N138Mfs*36) & (p.G96S) & & & & & & & \\
\hline \multirow[t]{2}{*}{$33^{*}$} & c. $286 G>A$ & c. $1000 C>T$ & $L P / L P$ & 30 & 27.2 & $1.97 \uparrow$ & $0.09 \uparrow$ & $1.99 \uparrow$ & (1)(2)(4) \\
\hline & (p.G96S) & (p.R344C) & & & & & & & \\
\hline \multirow[t]{2}{*}{34} & $c .286 G>A$ & c.1150G > C $\#$ & LPNUS & 30 & 21.3 & $1.04 \uparrow$ & $0.11 \uparrow$ & $1.24 \uparrow$ & (4) \\
\hline & (p.G96S) & (p.V384L) & & & & & & & \\
\hline \multirow[t]{2}{*}{35} & c. $473 A>G^{\#}$ & c. 413 del $A^{\#}$ & VUS/P & 31 & 23.4 & $1.82 \uparrow$ & $0.19 \uparrow$ & $2.28 \uparrow$ & (3)(4) \\
\hline & (p.Y158C) & (p.N138Mfs*36) & & & & & & & \\
\hline \multirow[t]{2}{*}{36} & c. $1000 C>T$ & c. $1092+1 G>A$ & $L P / P$ & 27.2 & 33 & $1.9 \uparrow$ & $0.1 \uparrow$ & $1.2 \uparrow$ & (4) \\
\hline & (p.R344C) & & & & & & & & \\
\hline \multirow[t]{2}{*}{37} & c. $286 G>A$ & c.500delG ${ }^{\#}$ & $L P / P$ & 30 & 25.3 & $1.61 \uparrow$ & $0.08 \uparrow$ & $1.46 \uparrow$ & - \\
\hline & (p.G96S) & $\left(p . S 167 M f s^{*} 7\right)$ & & & & & & & \\
\hline \multirow[t]{2}{*}{38} & c. $286 G>A$ & c.500delG ${ }^{\#}$ & LP/P & 30 & 25.3 & $1.43 \uparrow$ & $0.08 \uparrow$ & $0.48 \uparrow$ & (3)(4) \\
\hline & (p.G96S) & $\left(p . S 167 M_{f *}^{*} 7\right)$ & & & & & & & \\
\hline \multirow[t]{2}{*}{39} & c. $286 G>A$ & c. $286 G>A$ & LP/LP & 30 & 30 & $2.42 \uparrow$ & $0.07 \uparrow$ & $0.9 \uparrow$ & (4) \\
\hline & (p.G96S) & (p.G96S) & & & & & & & \\
\hline \multirow[t]{2}{*}{40} & c. $286 G>A$ & c. $1165 C>T^{\#}$ & LP/LP & 30 & 26.5 & $2.71 \uparrow$ & $0.13 \uparrow$ & $1.12 \uparrow$ & (4) \\
\hline & (p.G96S) & (p.R389W) & & & & & & & \\
\hline
\end{tabular}

Clinical symptom includes (1)growth delay, (2)motor delay, (3)elevated urine IBG, (4)abnormal other blood biochemical parameters; "-": normal

IBD isobutyryl-CoA dehydrogenase; NBS Newborn screening; $C 4$ isobutyl carnitine; $C 2$ acetyl carnitine; $C 3$ propionyl carnitine; $L P$ likely pathogenic; $P$ pathogenic; VUS variant uncertain significance; $L B$ likely benign

\# Novel variants in this study

33*, patient \#33 have a de novo variant c.2739del (p.E914Rfs*35) in KMT2A,

(Table 1). Twenty-nine (74\%) patients carried compound heterozygous variants, and $10(26 \%)$ patients carried homozygous variants. The predominant homoallelic genotype was [p. G96S]; [p. G96S], detected in 9 individuals, and the other one was [p. R392S]; p. R392S] in patient \#24. As shown in Fig. 1A, 25 variants were identified in our patients, including 4 splicing variants (16\%; c. 110 $2 \mathrm{~A}>\mathrm{T}$, c. $381-14 \mathrm{G}>\mathrm{A}$, c.842-1G $>\mathrm{A}, \mathrm{c} .1092+1 \mathrm{G}>\mathrm{A})$, a synonymous variant (c.444G $>$ T p. P148P), 16 missense variants $(64 \%)$, and 4 truncating variants $(16 \%)$ resulting from 4 deletions, c.4_5delCT (p. L2Vfs"40), c.413delA (p. N138Mfs*36), c.500delG (p. S167Mfs*7) and c.712delT 
(p. W238fs"9). Given its protein structure [17], most amino acid alterations caused by variants in IBD are localized in its catalytic domain (Fig. 1B), while two variants, p. L2Vfs" 40 and p. P37S are located in the mitochondria targeting sequence (MTS), and the p.S167Mfs*7 variant occurs in a binding domain for substrate FAD (flavin adenine dinucleotide). Notably, 16 novel variants were identified, including 10 missense variants (Fig. 1C): c.109C $>$ T (p. P37S), c.236G $>$ A (p. R79Q), c.259G > A (p. G87R), c.473A > G (p. Y158C), c.758 T > G (p. V253G), c.911A $>$ T (p. Q304L), c.989G >A (p. R330Q), c.1150G > C (p. V384L), c.1157A > G (p. Q386R), and $\mathrm{c} .1165 \mathrm{C}>\mathrm{T}$ (p. $\mathrm{R} 389 \mathrm{~W}) ; 3$ truncating variants:
c.4_5delCT (p. L2Vfs*40), c.413delA (p. N138Mfs*36) and c.500delG (p. S167Mfs*7); and 3 splicing variants described above.

According to ACMG recommendations (Table 1), three novel truncating variants (c.4_5delCT, c.413delA, c.500delG) and novel splicing variants (c.110-2A $>$ T, c.381-14G > A, c.842-1G >A) were postulated to be pathogenic $(\mathrm{P})$, as along with the reported pathogenic variant c. $455 \mathrm{~T}>\mathrm{C}$. The novel variant c.236G $>$ A alters an arginine to glutamine (p. R79Q), like the previously reported c.235C > G (p. R79G) variant [10]. Similarly, the novel variant c.989G>A changes the same amino acid, R330, which was previously reported in c.988C $>\mathrm{T}$

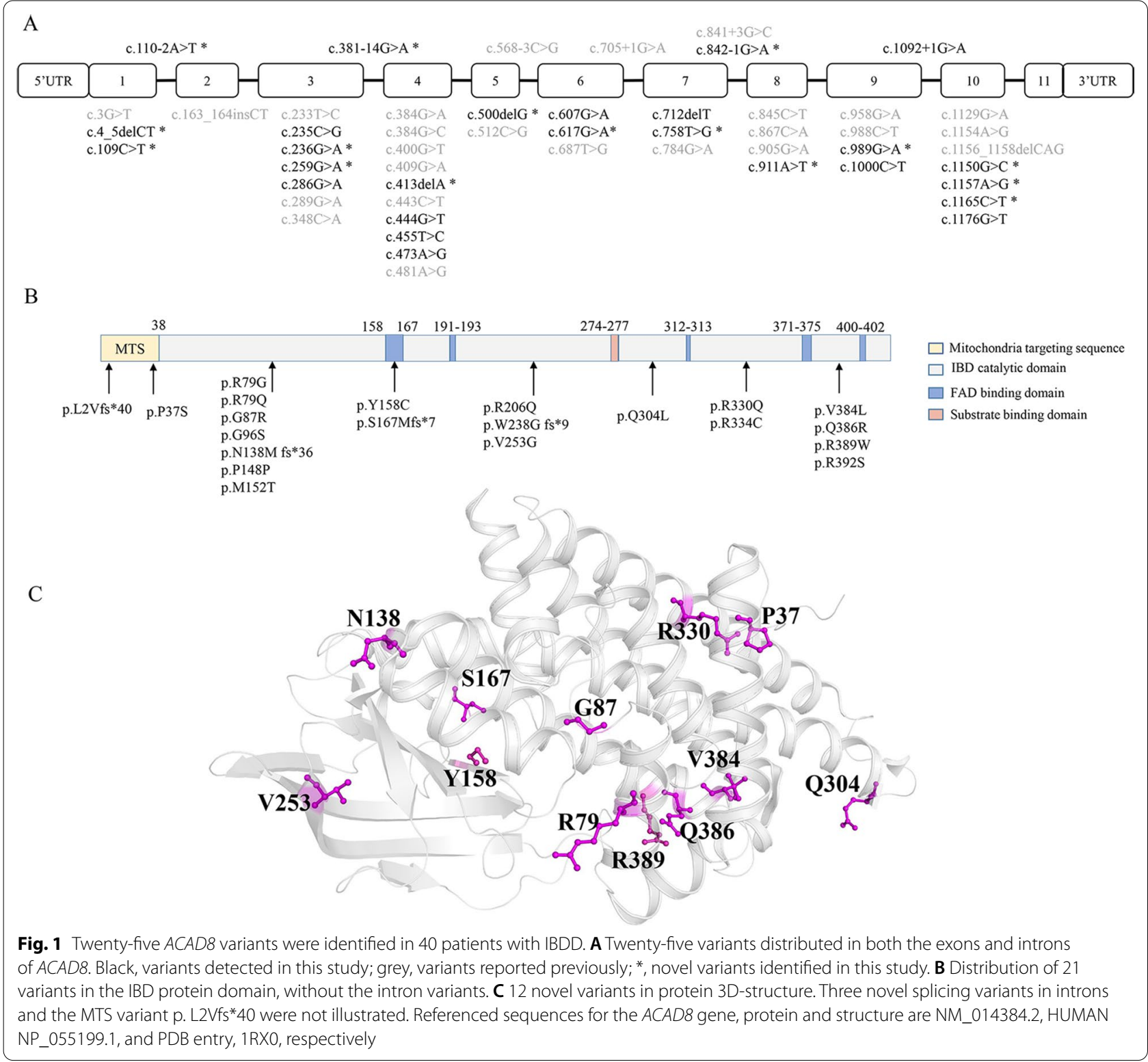


(R330W) [7]. As shown in Fig. 1B, both c.236G > A and c.989G $>$ A are localized in their catalytic domains, while c. $109 \mathrm{C}>\mathrm{T}$ is in the mitochondria-targeting sequence, which is crucial for protein imported into mitochondria. Novel missense variants c.911A $>$ T and c. $1157 \mathrm{~A}>\mathrm{G}$ were absent in the gnomAD database. Based on the evaluation using bioinformatics programs (Additional file 1: Table S1), all 10 novel missense variants were predicted as functional variants that would exert damaging effects on its protein.

The missense variant c.286G>A (p. G96S) was reported to be most common in the Chinese population, with an approximately frequency of 50\% [10], which occurred here in 26 individuals with an allelic frequency of $44 \%$, followed by c.1000C > T (p. R334C), c.1176G > T (p. R392S), c.413delA (p. N138Mfs*36) and c.500delG (p. S167Mfs"7) with allelic frequencies of $10 \%, 5 \%, 5 \%$ and $5 \%$, respectively. Most patients had their unique genotype. There were 27 genotypes distributed in 39 biallelic individuals. Except for the [c.286G >A];[ c.286G >A] genotype in 9 patients, there were 3 genotypes shared by more than two patients, followed as [c.286G $>\mathrm{A}]$; [c. $1000 \mathrm{C}>\mathrm{T}$ ] by 3 patients (P\#11, \#12 and \#33), [c.286G > A]; [c.413delA] by 2 patients (P\#15 and \#32), and [c.286G >A]; [c.500delG] by 2 patients (P\#37 and $\# 38$ ).

\section{Review of $A C A D 8$ variants in IBDD}

Including our patient, 87 individuals with IBDD have been described $[1-7,9-23]$, of which 81 underwent genetic testing for $A C A D 8$ and 78 individuals were confirmed to have biallelic variants. Fifty-one types of variants were detected, including $36(70 \%)$ missense variants, one nonsense variant $(2 \%, c .348 \mathrm{C}>\mathrm{A})$, one synonymous variant $(2 \%$, c. $444 \mathrm{G}>\mathrm{T}), 7$ (14\%) splicing sites between introns and exon barriers and $6(12 \%)$ truncating variants. As shown in Fig. 2A, only 3 variants, c.289G $>$ A, c.455 $\mathrm{T}>\mathrm{C}$ and c.1000 $\mathrm{C}>\mathrm{T}$, occurred in both Asian and Caucasian population. To date, the variant c.286 G>A was most prevalent in IBDD, specifically present in 44 Chinese patients $[10,19]$, followed by variants $c$. $1000 \mathrm{C}>\mathrm{T}$ in 14 patients, c.1176G $>\mathrm{A}$ in 6 patients and $\mathrm{c}$.

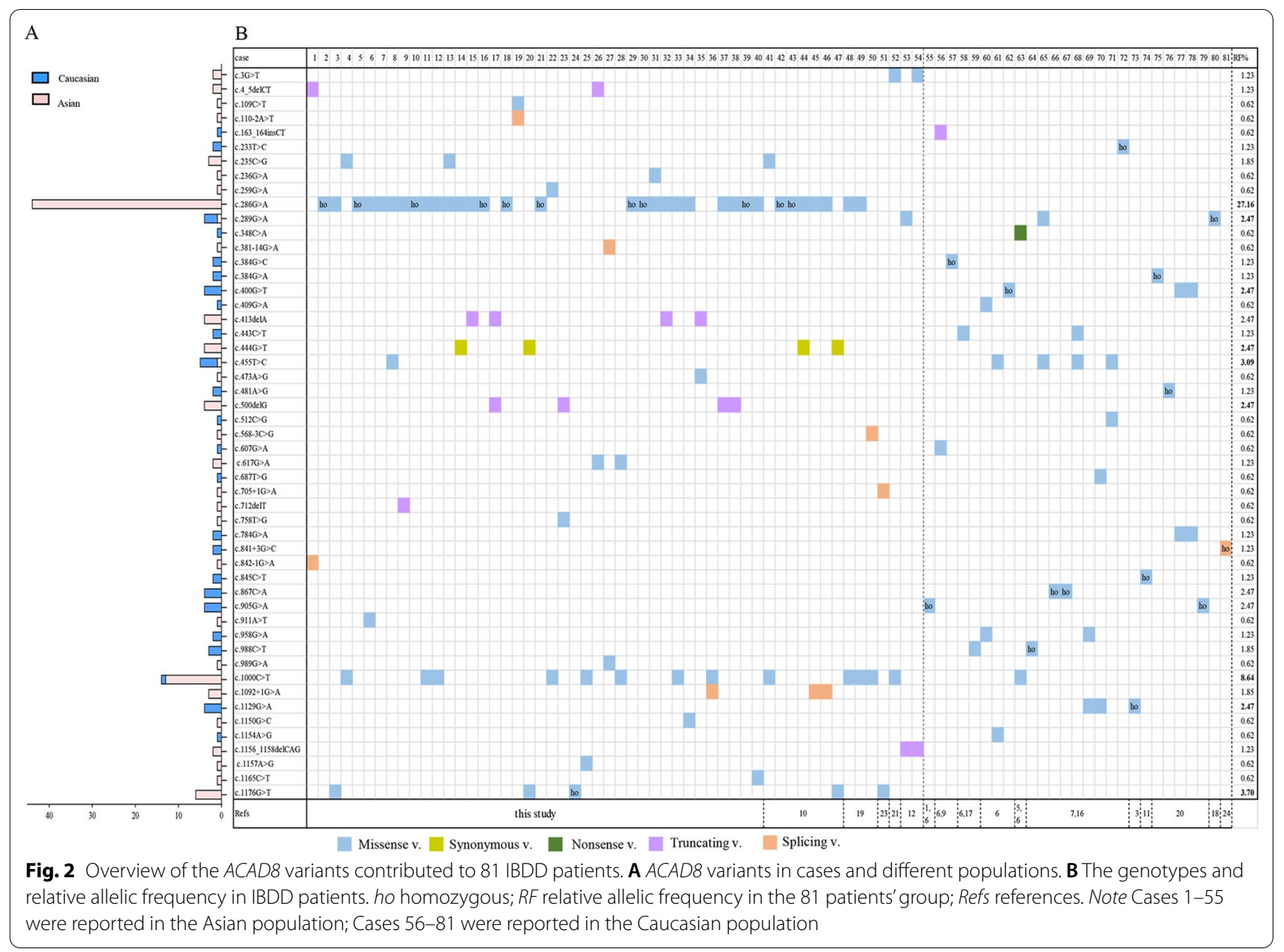


$455 \mathrm{~T}>\mathrm{C}$ in 5 patients. There were 57 different genotypic combinations in 78 patients. Consistent with our finding, $67 \%$ of cases with biallelic variants were predominantly genotyped with compound heterozygous alleles (Fig. 2B). However, only 26 IBDD individuals carried homozygous alleles (Fig. 2B). Similarly, most patients $(62 \%, 48 / 78)$ had their unique genotype. However, 9 different genotypic combinations were shared in 30 individuals, including [c.286G > A];[c.286G >A] in 9 patients and [c.286G >A]; [c. $1000 \mathrm{C}>\mathrm{T}$ ] in 5 patients (Fig. $2 \mathrm{~B}$ ).

\section{Discussion}

IBDD is a rare metabolic disorder that is less well understood, as only dozens of cases have been described previously in the literature [10-12]. Due to the limited number of cases, the IBDD prevalence varies substantially in different regions and/or populations. Here, based on the NBS program and clinical diagnosis, 40 patients with IBDD were identified with a prevalence of $1 / 62,599$ in Zhejiang Province, which is close to the incidence $(1 / 70,000)$ estimated by Oglesbee et al. [7], but higher than the incidence reported in California $(1 / 292,451)$ by Gallant et al. [4]. Consistent with published cases, most patients with IBDD turned out healthy and had no clinical sequelae. Clinical symptoms, including increased urinary IBG levels, mild delay in growth and development, were generally transient during follow-up and had been restored by dietary management. However, there was no direct correlation between the improvement of clinical outcomes (including growth-developmental delay and impaired liver function) and diet, and several developmental delay cases followed an age-appropriate intake of calories and protein after 6 months of age, similar to other children. Nevertheless, elevated C4-acylcarnitine concentrations always exist. Neonatal hypoglycemia in patients with IBDD that reported elsewhere was absent here [20], while 10 patients had anaemia instead. However, Acad 8 mutant mice showed significantly elevated transaminase levels and presented progressive hepatic steatosis [24]. Strikingly, some biochemical indicators associated with liver function were aberrant in 17 patients during follow-up. Regarding anaemia or transient liver lesions, we are still unaware whether those clinical symptoms are in connection with IBDD. A few patients had developmental delay, speech delay or hypotonia $[10,12]$. Unfortunately, patient \#33 presented a severe delay in both growth and development with an inflexible increase in serum C4-acylcarnitine levels, which was further genetically confirmed to be a novel variant, c.2739del (p. E914Rfs*35), in KMT2A that associated with WDSTS. Another special IBDD patient, with a severe lack of speech development and lack of social interactions, was reported to be associated with autism [18]. Therefore, appropriate interventions are required once IBDD is diagnosed, and patients with IBDD should be monitored carefully. All patients received proper dietary interventions as stated and had a positive prognosis during clinical monitoring.

Both the genotypes and $A C A D 8$ variants in IBDD are highly heterogeneous. A total of 51 variants were reported in 81 IBDD patients and were widely distributed along the gene. More than $60 \%$ of patients presented with a unique genotype, and the compound heterozygotes were predominant. The variants c.286G $>\mathrm{A}$ (p. G96S), c.1000C $>$ T (p. R334C), c.1176G > A (p. R392S) and c.455 T>C (p. M152T) appeared to be more prevalent in 81 IBDD patients, with relative frequencies of $27.2 \%, 8.6 \%, 3.7 \%$ and $3.1 \%$ (Fig. 2B). To date, the hotspot variant, c.286G $>$ A has been observed solely in Chinese patients [10, 19], indicating that it is specific in this population and needs more cases to be confirmed. In this study, 16 ACAD8 variants were first reported in IBDD patients. It should be certain that more novel variants will be detected in future cases with the widespread utilization of genetic testing in IBDD. The genotype-phenotype correlation was unclear here. Nine patients with the same genotype, [c.286G >A]; [c.286G >A], presented mild symptoms or were asymptomatic with different biochemical indicators (Additional file 1: Table 2). On the other hand, 8 patients with elevated urinary IBG carried different genotypes, including $\mathrm{LP} / \mathrm{LP}, \mathrm{LP} / \mathrm{P}$ or $\mathrm{P} /$ VUS combination (Table 1). Intriguingly, Patients \#14 and $\# 20$, harboring a synonymous variant c.444G $>\mathrm{T}$ (p. P148P) with a low CADD score [25], had a normal level of $\mathrm{C} 4 / \mathrm{C} 2(0.05)$ at newborn screening and occasionally elevated to 0.08 during follow-up. Patient \#17, with 2 deletions, potentially no IBD activity, had moderate elevations of $\mathrm{C} 4$ without a phenotype.

In summary, up to 40 patients with IBDD were diagnosed with a prevalence of 1/62,597 in Zhejiang Province. IBDD patients picked up by NBS have a mild phenotype here. Most patients were healthy during follow-up, except one who was associated with WDSTS. Sixteen novel $A C A D 8$ variants were identified. Based on a review of the variant spectrum in IBDD, we found that c. $286 \mathrm{G}>\mathrm{A}$ and $\mathrm{c} .1000 \mathrm{C}>\mathrm{T}$ were prevalent in patients, of which c. $286 \mathrm{G}>\mathrm{A}$ has been observed solely in the Chinese population. Similar to other metabolic disorders, compound heterozygotes were predominant in genotypes. No clear genotype-phenotype correlation existed in IBDD patients. Here, our IBDD cohort with detailed clinical characteristics, genotypes and 
long-term prognosis will be helpful for the diagnosis and management of patients with IBDD.

\section{Conclusions}

Isobutyryl-CoA dehydrogenase deficiency is a rare metabolic disorder with an incidence of 1/62,599 in Zhejiang Province. The concentration of C4-acylcarnitine in NBS plus subsequent genetic testing is helpful for IBDD diagnosis. Both the genotypes and ACAD8 variants in IBDD are highly heterogeneous, No clear correlation between genotype and phenotype is presented here in patients with IBDD.

\section{Supplementary Information}

The online version contains supplementary material available at https://doi. org/10.1186/s13023-021-02018-6.

Additional file 1: Table 1. redicted functional effects of 10 novel missense variants; Table 2 . Clinical features and genotypes of 40 IBDD patients.

\section{Acknowledgements}

We are grateful to all patients and their family members for their participation.

\section{Authors' contributions}

Research idea and study design: FT, PJ and QS; patient management and clinical evaluation: QS, FT, LZ, and CC; data analysis and interpretation: JF, CY, $L Z, Y Z, X Z$, and $Q C$; drafting the paper: JF and $C Y$; primary responsibility to the final version of the manuscript: FT, PJ and QS; All authors reviewed the manuscript. All authors read and approved the final manuscript.

\section{Funding}

The work was supported by the National Natural Science Foundation of China under Grant \#81870314 to P.J., the National Key Technologies R\&D Program under Grant \#2017YFC1001700 to Q.S and F.T., and the Zhejiang Provincial Program for the Cultivation of High-level Innovative Health talents to P.J.

\section{Availability of data and materials}

The dataset supporting the conclusions of this article is included within the article and its supplementary information files.

\section{Declarations}

\section{Ethics approval and consent to participate}

All procedures followed were in accordance with approval from the Research Ethics Committees, the Children's Hospital of Zhejiang University School of Medicine. Written informed consent was obtained from the patients/participants for inclusion in the study.

\section{Consent for publication}

Not applicable.

\section{Competing interests}

The authors declare no competing interests.

\section{Author details}

${ }^{1}$ Department of Genetic and Metabolic Disease, The Children's Hospital, Zhejiang University School of Medicine, National Clinical Research Center for Child Health, 3333 Binsheng Road, Hangzhou 310052, China. ${ }^{2}$ Institute of Genetics and Department of Human Genetics, Zhejiang University School of Medicine, Hangzhou 310058, China. ${ }^{3}$ Zhejiang Provincial Key Lab of Genetic and Developmental Disorders, Hangzhou 310058, China.
Received: 15 July 2021 Accepted: 13 September 2021

Published online: 20 September 2021

\section{References}

1. Roe CR, Cederbaum SD, Roe DS, Mardach R, Galindo A, Sweetman L. Isolated isobutyryl-CoA dehydrogenase deficiency: an unrecognized defect in human valine metabolism. Mol Genet Metab. 1998;65(4):264-71. https://doi.org/10.1006/mgme.1998.2758.

2. Nguyen TV, Andresen BS, Corydon TJ, Ghisla S, Abd-El Razik N, Mohsen A-WA, et al. Identification of isobutyryl-CoA dehydrogenase and its deficiency in humans. Molec Genet Metab. 2002; 77: 68-79. https://doi.org/ 10.1016/S1096-7192(02)00152-X.

3. Scolamiero E, Cozzolino C, Albano L, Ansalone A, Caterino M, Corbo G, et al. Targeted metabolomics in the expanded newborn screening for inborn errors of metabolism. Mol Biosyst. 2015;11(6):1525-35. https://doi. org/10.1039/c4mb00729h.

4. Gallant NM, Leydiker K, Tang H, Feuchtbaum L, Lorey F, Puckett R, et al. Biochemical, molecular, and clinical characteristics of children with short chain acyl-CoA dehydrogenase deficiency detected by newborn screening in California. Mol Genet Metab. 2012;106:55-61. https://doi.org/10. 1016/j.ymgme.2012.02.007

5. Koeberl DD, Young SP, Gregersen NS, Vockley J, Smith WE, Benjamin DK Jr, et al. Rare disorders of metabolism with elevated butyryl- and isobutyrylcarnitine detected by tandem mass spectrometry newborn screening. Pediatr Res. 2003;54(2):219-23. https://doi.org/10.1203/01.PDR.00000 74972.36356 .89$.

6. Pedersen CB, Bischoff C, Christensen E, Simonsen H, Lund AM, Young SP, et al. Variations in IBD (ACAD8) in children with elevated C4-carnitine detected by tandem mass spectrometry newborn screening. Pediatr Res. 2006;60:315-20. https://doi.org/10.1203/01.pdr.0000233085.72522.04.

7. Oglesbee D, He M, Majumder N, Vockley J, Ahmad A, Angle B, et al. Development of a newborn screening follow-up algorithm for the diagnosis of isobutyryl-CoA dehydrogenase deficiency. Genet Med. 2007;9(2):108-16. https://doi.org/10.1097/gim.0b013e31802f78d6.

8. Minkler PE, Stoll MS, Ingalls ST, Kerner J, Hoppel CL. Quantitative acylcarnitine determination by UHPLC-MS/MS-Going beyond tandem MS acylcarnitine "profiles." Mol Genet Metab. 2015;116(4):231-41. https://doi. org/10.1016/j.ymgme.2015.10.002.

9. Sass JO, Sander S, Zschocke J. Isobutyryl-CoA dehydrogenase deficiency: isobutyrylglycinuria and ACAD8 gene mutations in two infants. J Inherit Metab Dis. 2004;27(6):741-5. https://doi.org/10.1023/B:BOLI.0000045798. $12425.1 \mathrm{~b}$.

10. Lin Y, Peng W, Jiang M, Lin C, Lin W, Zheng Z, et al. Clinical, biochemical and genetic analysis of Chinese patients with isobutyryl-CoA dehydrogenase deficiency. Clin Chim Acta. 2018;487:133-8. https://doi.org/10. 1016/j.cca.2018.09.033.

11. Santra S, Macdonald A, Preece MA, Olsen RK, Andresen BS. Long-term outcome of isobutyryl-CoA dehydrogenase deficiency diagnosed following an episode of ketotic hypoglycaemia. Mol Genet Metab Rep. 2016;10:28-30. https://doi.org/10.1016/j.ymgmr.2016.11.005.

12. Yun JW, Jo KI, Woo HI, Lee SY, Ki CS, Kim JW, Park HD. A novel ACAD8 mutation in asymptomatic patients with isobutyryl-CoA dehydrogenase deficiency and a review of the ACAD8 mutation spectrum. Clin Genet. 2015;87(2):196-8. https://doi.org/10.1111/cge.12350.

13. Nygaard $H$, Gaist D, Christensen M, Dunø M, Kjeldsen M, Schrøder H, et al. Isobutyryl-CoA dehydrogenase deficiency presenting with significant clinical disease in adulthood, The 18th Nordic Congress in Human Genetics. Reykjavik, Island, 2016.

14. Knerr I, Weinhold N, Vockley J, Gibson KM. Advances and challenges in the treatment of branched-chain amino/keto acid metabolic defects. J Inherit Metab Dis. 2012;35(1):29-40. https://doi.org/10.1007/ s10545-010-9269-1.

15. Richards S, Aziz N, Bale S, Bick D, Das S, Gastier-Foster J, et al. Standards and guidelines for the interpretation of sequence variants: a joint consensus recommendation of the American College of Medical Genetics and Genomics and the Association for Molecular Pathology. Genet Med. 2015;17:405-24. https://doi.org/10.1038/gim.2015.30. 
16. Pena L, Angle B, Burton B, Charrow J. Follow-up of patients with shortchain acyl-CoA dehydrogenase and isobutyryl-CoA dehydrogenase deficiencies identified through newborn screening: one center's experience. Genet Med. 2012;14(3):342-7. https://doi.org/10.1038/gim.2011.9.

17. Battaile KP, Nguyen TV, Vockley J, Kim JP. Structures of isobutyryl-CoA dehydrogenase and enzyme-product complex: comparison with isovaleryl- and short-chain acyl-CoA dehydrogenases. J Biol Chem. 2004;279(16):16526-34. https://doi.org/10.1074/jbc.M400034200.

18. Eleftheriadou M, Medici-van den Herik E, Stuurman $K$, van Bever $Y$, Hellebrekers DMEI, van Slegtenhorst M, et al. Isobutyryl-CoA dehydrogenase deficiency associated with autism in a girl without an alternative genetic diagnosis by trio whole exome sequencing: A case report. Mol Genet Genomic Med. 2021; 9(2):1595. https://doi.org/10.1002/mgg3.1595.

19. Wang T, Ma J, Zhang Q, Gao A, Wang QI, Li H, et al. Expanded newborn screening for inborn errors of metabolism by tandem mass spectrometry in Suzhou, China: disease spectrum, prevalence, genetic characteristics in a chinese population. Front Genet. 2019;10:1052. https://doi.org/10.3389/ fgene.2019.01052.

20. Sadat R, Hall PL, Wittenauer AL, Vengoechea ED, Park K, Hagar AF, et al. Increased parental anxiety and a benign clinical course: Infants identified with short-chain acyl-CoA dehydrogenase deficiency and isobutyryl-CoA dehydrogenase deficiency through newborn screening in Georgia. Mol Genet Metab. 2020;129(1):20-5. https://doi.org/10.1016/j.ymgme.2019. 11.008.

21. Yoo EH, Cho HJ, Ki CS, Lee SY. Isobutyryl-CoA dehydrogenase deficiency with a novel ACAD8 gene mutation detected by tandem mass spectrometry newborn screening. Clin Chem Lab Med. 2007:45(11):1495-7. https://doi.org/10.1515/CCLM.2007.317.

22. Wang W, Yang J, Xue J, Mu W, Zhang X, Wu W, et al. A comprehensive multiplex PCR based exome-sequencing assay for rapid bloodspot confirmation of inborn errors of metabolism. BMC Med Genet. 2019;20(1):3. https://doi.org/10.1186/s12881-018-0731-5.

23. Popek M, Walter M, Fernando M, Lindner M, Schwab KO, Sass JO. Two inborn errors of metabolism in a newborn: glutaric aciduria type I combined with isobutyrylglycinuria. Clin Chim Acta. 2010;411:2087-91. https://doi.org/10.1016/j.cca.2010.09.006.

24. Sabbagha NG, Kao HJ, Yang CF, Huang CC, Lin WD, Tsai FJ, et al. Alternative splicing in Acad8 resulting a mitochondrial defect and progressive hepatic steatosis in mice. Pediatr Res. 2011;70(1):31-6. https://doi.org/10. 1203/PDR.0b013e31821b89ee.

25. Kircher M, Witten DM, Jain P, O'Roak BJ, Cooper GM, Shendure J. A general framework for estimating the relative pathogenicity of human genetic variants. Nat Genet. 2014;46(3):310-5. https://doi.org/10.1038/ng.2892.

\section{Publisher's Note}

Springer Nature remains neutral with regard to jurisdictional claims in published maps and institutional affiliations.

Ready to submit your research? Choose BMC and benefit from:

- fast, convenient online submission

- thorough peer review by experienced researchers in your field

- rapid publication on acceptance

- support for research data, including large and complex data types

- gold Open Access which fosters wider collaboration and increased citations

- maximum visibility for your research: over $100 \mathrm{M}$ website views per year

At BMC, research is always in progress.

Learn more biomedcentral.com/submissions 